\title{
LOW MASSES AND HIGH REDSHIFTS: THE EVOLUTION OF THE MASS-METALLICITY RELATION*
}

\author{
Alaina Henry ${ }^{1,2}$, Claudia Scarlata ${ }^{3}$, Alberto Domínguez $^{4}$, Matthew Malkan $^{5}$, Crystal L. Martin $^{2}$, Brian Siana ${ }^{4}$, \\ Hakim AteK $^{6}$, Alejandro G. Bedregal ${ }^{3,7}$, James W. Colbert ${ }^{8}$, Marc Rafelski $^{8}$, Nathaniel Ross ${ }^{5}$, Harry Teplitz ${ }^{9}$, \\ Andrew J. Bunker ${ }^{10}$, Alan Dressler ${ }^{11}$, Nimish Hathi ${ }^{11}$, Daniel Masters ${ }^{4}, 11$, Patrick McCarthy ${ }^{11}$, and Amber Straughn ${ }^{1}$ \\ ${ }^{1}$ Astrophysics Science Division, Goddard Space Flight Center, Code 665, Greenbelt, MD 20771, USA; alaina.henry@ nasa.gov \\ ${ }^{2}$ Department of Physics, University of California, Santa Barbara, CA 93106, USA \\ ${ }^{3}$ Minnesota Institute for Astrophysics, University of Minnesota, Minneapolis, MN 55455, USA \\ ${ }^{4}$ Department of Physics and Astronomy, University of California, Riverside, Riverside, CA 92521, USA \\ ${ }^{5}$ Department of Physics and Astronomy, University of California, Los Angeles, Los Angeles, CA 90095, USA \\ ${ }^{6}$ Laboratoire d'astrophysique, École Polytechniuqe Fédérale de Lausanne, Observatoire de Sauverny, 1290 Versoix, Switzerland \\ 7 Department of Physics and Astronomy, Tufts University, Medford, MA 02155, USA \\ ${ }^{8}$ Spitzer Science Center, California Institute of Technology, Pasadena, CA 91125, USA \\ ${ }^{9}$ Infrared Processing and Analysis Center, Caltech, Pasadena, CA 91125, USA \\ ${ }^{10}$ Department of Physics, University of Oxford, Denys Wilkinson Building, Keble Road, OX1 3RH, UK \\ ${ }^{11}$ Observatories of the Carnegie Institution for Science, Pasadena, CA 91101, USA \\ Received 2013 August 22; accepted 2013 September 16; published 2013 October 4
}

\begin{abstract}
We present the first robust measurement of the high redshift mass-metallicity (MZ) relation at $10^{8} \lesssim M / M_{\odot} \lesssim 10^{10}$, obtained by stacking spectra of 83 emission-line galaxies with secure redshifts between $1.3 \lesssim z \lesssim 2.3$. For these redshifts, infrared grism spectroscopy with the Hubble Space Telescope Wide Field Camera 3 is sensitive to the $R_{23}$ metallicity diagnostic: ([O II] $\left.\lambda \lambda 3726,3729+[\mathrm{O} \mathrm{III}] \lambda \lambda 4959,5007\right) / \mathrm{H} \beta$. Using spectra stacked in four mass quartiles, we find a MZ relation that declines significantly with decreasing mass, extending from $12+\log (\mathrm{O} / \mathrm{H})=$ 8.8 at $M=10^{9.8} M_{\odot}$, to $12+\log (\mathrm{O} / \mathrm{H})=8.2$ at $M=10^{8.2} M_{\odot}$. After correcting for systematic offsets between metallicity indicators, we compare our MZ relation to measurements from the stacked spectra of galaxies with $M \gtrsim 10^{9.5} M_{\odot}$ and $z \sim 2.3$. Within the statistical uncertainties, our MZ relation agrees with the $z \sim 2.3$ result, particularly since our somewhat higher metallicities (by around $0.1 \mathrm{dex}$ ) are qualitatively consistent with the lower mean redshift $(z=1.76)$ of our sample. For the masses probed by our data, the MZ relation shows a steep slope which is suggestive of feedback from energy-driven winds, and a cosmological downsizing evolution where high mass galaxies reach the local MZ relation at earlier times. In addition, we show that our sample falls on an extrapolation of the star-forming main sequence (the SFR- $M_{*}$ relation) at this redshift. This result indicates that grism emission-line selected samples do not have preferentially high star formation rates (SFRs). Finally, we report no evidence for evolution of the mass-metallicity-SFR plane; our stack-averaged measurements show excellent agreement with the local relation.
\end{abstract}

Key words: galaxies: abundances - galaxies: evolution

Online-only material: color figures

\section{INTRODUCTION}

The correlation between stellar mass and gas-phase metallicity in galaxies (the mass-metallicity or MZ relation) is sensitive to the processes that regulate their growth. This relation is shaped by gas outflow and accretion rates, the enrichment of these gas flows, and the star formation efficiency of galaxies (Tremonti et al. 2004; Brooks et al. 2007; Dalcanton 2007; Erb 2008; Finlator \& Davé 2008; Peeples \& Shankar 2011; Davé et al. 2012; Henry et al. 2013). It is expected, then, that observations of the MZ relation toward low masses and high redshifts will constrain the assembly of galaxies and the metal enrichment of the intergalactic medium.

To date, a complete picture of metallicity evolution has not emerged. While studies focusing on higher mass galaxies at $0.5<z<3$ have shown lower metallicities than are seen locally (Zahid et al. 2011; Shapley et al. 2005; Erb et al. 2006; Liu et al. 2008; Maiolino et al. 2008; Wright et al. 2009; Hayashi et al. 2009), evolution below $M \sim 10^{9.0}-10^{9.5} M_{\odot}$

\footnotetext{
* Based on observations made with the NASA/ESA Hubble Space Telescope, which is operated by the Association of Universities for Research in Astronomy, Inc., under NASA contract NAS 5-26555.
}

remains unconstrained. In Henry et al. (2013), we began to probe masses just below $M \sim 10^{8.5} M_{\odot}$ at intermediate redshifts; nevertheless measuring metallicities at higher redshifts $(z>1)$ has been challenging because of the requirement for infrared spectroscopy of large, faint samples of galaxies. Although lower masses and metallicities have been reached for some strongly lensed galaxies (e.g., Teplitz et al. 2000; Hainline et al. 2009; Wuyts et al. 2012; Brammer et al. 2012; Yuan et al. 2013; Belli et al. 2013), the uncertainties of these measurements are large.

In this Letter, we present the MZ relation at $z>1.3$, derived by stacking 83 galaxies with observations covering the $R_{23}$ metallicity diagnostic ([O II] $\lambda \lambda 3726,3729+[\mathrm{O}$ III] $\lambda \lambda 4959,5007) / \mathrm{H} \beta$ (Pagel et al. 1979). Our sample, which is drawn from Hubble Space Telescope Wide Field Camera 3 (WFC3; MacKenty et al. 2010) grism observations, reaches $M \sim 10^{8} M_{\odot}$ at $1.3<z<2.3$. By focusing on measurements from stacked spectra, we avoid bias introduced by the requirement that $\mathrm{H} \beta$ be detected in each spectrum. We adopt a Chabrier (2003) initial mass function (IMF) and take $\Omega_{M}=0.3$, $\Omega_{\Lambda}=0.7$ and $H_{0}=70 \mathrm{~km} \mathrm{~s}^{-1} \mathrm{Mpc}^{-1}$. When reporting measurements of doublet lines (i.e., [O III] $\lambda \lambda 4959,5007$ ), we use "[O II ]" or "[O III]" to refer to both lines. 


\section{OBSERVATIONS AND SAMPLE SELECTION}

We use data from the WFC3 Infrared Spectroscopic Parallel (WISP) Survey, as described by Atek et al. (2010, 2011), Domínguez et al. (2013), Colbert et al. (2013), and Bedregal et al. (2013). The WISP Survey uses both the G102 $(0.8-1.1 \mu \mathrm{m}$, $R \sim 210)$ and G141 (1.1-1.7 $\mu \mathrm{m}, R \sim 130)$ grisms, enabling measurements of $R_{23}$ from $1.3<z<2.3$. As the WISP survey is ongoing, we restrict our analysis to 29 fields that have emission-line catalogs and supporting optical imaging, either from WFC3/UVIS (24 fields) or from the Large Format Camera (Simcoe et al. 2000) on the 200-inch Hale Telescope at Palomar Observatory ( $g$ and $i$ bands; 5 fields). Five-band galaxy spectral energy distributions (SEDs; including Spitzer/IRAC $3.6 \mu \mathrm{m}$ photometry for most) are obtained from photometric catalogs described in Domínguez et al. (2013) and Bedregal et al. (2013).

Using the WISP emission line catalogs (Colbert et al. 2013), we identify 128 galaxies with secure redshifts measured from multiple lines. Objects with contamination from overlapping spectra were removed, leaving 74 galaxies. Of these, we exclude five galaxies which have inadequate mass constraints: three fall in the UVIS chip gap, one is detected in only one broad-band filter, and one is blended with another object in ground-based $g$ and $i$ images. These cuts leave 69 galaxies from WISP.

We supplement this sample with publicly released grism data covering the Ultra Deep Field (UDF); ${ }^{12}$ since the UDF is observed only in G141, $R_{23}$ is covered only for the 25 galaxies between $2.04<z<2.36$. Of these, we exclude three galaxies because of contamination, two because their spectra do not contain emission lines, and one with incomplete imaging near the edge of the UDF. We construct SEDs for the remaining 19 galaxies using the nine bands of imaging in the HST eXtreme Deep Field (Illingworth et al. 2013). Photometric catalogs were created by using SExtractor (Bertin \& Arnouts 1996) in dual image mode, with $\mathrm{F} 160 \mathrm{~W}$ as the detection image. We use MAG_AUTO to estimate the total flux. Errors are increased to account for correlated noise in drizzled data (Casertano et al. 2000).

Among the 88 galaxies in the combined sample, we identify and remove five active galactic nucleus (AGN) candidates (Section 3.3). The mean redshift for the remaining 83 galaxies is $z=1.76$.

\section{MEASUREMENTS}

\subsection{Emission Line Fluxes}

We measure emission line fluxes from the individual spectra by simultaneously fitting Gaussian profiles to the [O II], [O III], and $\mathrm{H} \beta$ lines, as well as $\mathrm{H} \alpha$ and [S II] when they are covered. Contribution to $\mathrm{H} \alpha$ from unresolved [N II] $\lambda \lambda 6548,6583$ is taken to be negligible, consistent with our followup spectroscopy (D. Masters et al., in preparation). Each region of the spectrum (i.e., [O II $],[\mathrm{O} I I I]+\mathrm{H} \beta, \mathrm{H} \alpha+\left[\mathrm{S}_{\mathrm{II}}\right]$ ) is also fit with a linear continuum. Because the dispersion in the grism spectra diverges from the adopted solution at the red and blue ends, we allow for shifts of up to $\Delta z= \pm 0.01$ between the three widely separated groups of emission lines. We take the redshift from the fit to [O III] and $\mathrm{H} \beta$. Additionally, since the [O III $] \lambda \lambda 4959,5007$ doublet is marginally resolved, we fit both lines assuming a 3:1 flux ratio. Finally, the emission lines should be spectrally unresolved with widths set by the size of the galaxy in the dispersion

\footnotetext{
12 http://monoceros.astro.yale.edu/RELEASE_V3.0/Spectra/UDF/Web/
} UDF_3dhst_redshift_v1.0.html

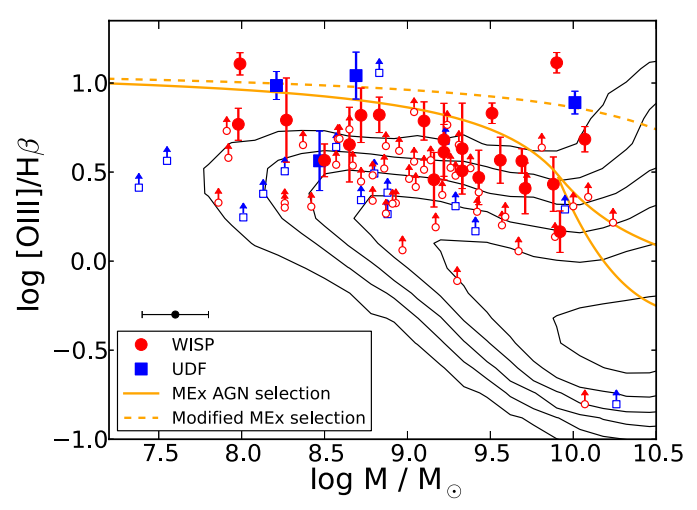

Figure 1. The MEx diagram shows that a minority of WISP and UDF emission line objects are AGN. Filled symbols show the objects where $\mathrm{H} \beta$ emission is detected at $3 \sigma$ significance or better ( 25 galaxies). Open symbols represent $3 \sigma$ limits (63 galaxies). The typical stellar mass uncertainty is shown in the lower left. Contours show the local relation, derived from SDSS DR7 catalogs (http://www.mpa-garching.mpg.de/SDSS). Orange lines show AGN thresholds. (A color version of this figure is available in the online journal.)

direction. Since the dispersion is two times higher in the G102 spectra, we require lines measured in this grism to have widths a factor of two narrower (in $\AA$ ) than those of the redder lines.

\subsection{Stellar Mass}

Stellar masses are derived with the FAST software (Kriek et al. 2009), fitting BC03 (Bruzual \& Charlot 2003) template spectra to our broadband photometry. We take a grid of stellar population parameters that include: exponentially declining star formation histories with $e$-folding times ranging from $40 \mathrm{Myr}$ to $10 \mathrm{Gyr}$; ages ranging from $50 \mathrm{Myr}$ to the age of the universe, and $A_{V}=0-3$ for a Calzetti et al. (2000) extinction curve. Additionally, we use a Chabrier (2003) IMF with metallicities of $0.004,0.008$, and $0.02\left(Z_{\odot}\right)$. We removed the emission line contribution to our broadband photometry, estimating the corrections by integrating our continuum subtracted spectra (see Section 3.4) under the bandpasses that cover the line emission.

\section{3. $A G N$}

In Figure 1, we use the Mass Excitation (MEx) diagram to identify AGN that could contaminate our stacked spectra. This diagnostic uses mass as a proxy for the $\left[\mathrm{N}_{\mathrm{II}}\right] \lambda 6583 / \mathrm{H} \alpha$ ratio, which is not measured for our sample. The solid orange lines show the AGN threshold from Juneau et al. (2011); objects above and to the right are classified as AGN, and objects between the solid curves at $M \gtrsim 10^{10} M_{\odot}$ are star-forming/ AGN composites. However, the use of the MEx diagnostic is compromised by the evolution of the $\mathrm{MZ}$ relation. At fixed metallicity (or $[\mathrm{O} \mathrm{III}] / \mathrm{H} \beta$ ), galaxies at $z \sim 1-2$ are $1-2 \mathrm{dex}$ more massive than $z \sim 0$ galaxies (Shapley et al. 2005; Erb et al. 2006). Therefore, we expect that the star-forming locus in Figure 1 shifts to the right at higher redshifts. The dashed curve illustrates the possible evolution, by shifting the Juneau et al. curves a conservative $1 \mathrm{dex}$ in stellar mass. Taking this modified threshold, we find five AGN candidates. These objects are excluded from further analysis. We acknowledge that the more aggressive AGN selection would slightly modify our MZ relation above $M>10^{9.4} M_{\odot}$, increasing the metallicity derived in Section 3.5 by $0.03 \mathrm{dex}$ at $M=10^{9.8} M_{\odot}$. Nevertheless, this shift falls within our uncertainties, and does not affect the conclusions drawn in Sections 4 and 5. 
For the galaxies that are undetected in $\mathrm{H} \beta$, we cannot rule out an AGN for any given object. However, given the distribution of detections and limits in Figure 1, it is unlikely that a sizable fraction of these galaxies have very high $[\mathrm{O} \mathrm{III}] / \mathrm{H} \beta$ ratios. We also note that none of the UDF galaxies are identified as AGN from X-ray counterparts in the Xue et al. (2011) catalog. Therefore, we conclude that stacked spectra will be minimally affected by this contamination. Overall, the low AGN content in our sample is consistent with the low mass, grism-selected objects reported by Trump et al. (2013).

\subsection{Spectral Stacking}

In order to stack spectra, we follow a procedure similar to Domínguez et al. (2013). First, we subtract a model continuum for each galaxy, made by masking the emission lines and smoothing with a 30 pixel boxcar. Each spectrum is then normalized by its [O III] flux, so that all galaxies contribute equally. Finally, the spectra are de-redshifted and combined by taking the median. The relative line fluxes are determined by fitting Gaussian profiles. The measurement follows the same procedure described in Section 3.1, except the widths of the emission lines are allowed to differ from one another because the relative contribution of the G102 and G141 data varies with restwavelength. Error spectra are generated by bootstrap resampling the spectra in each mass bin to generate 500 artificial stacks, and taking the rms at each wavelength. Errors on the relative line fluxes are calculated by propagating the uncertainties on the fit parameters.

Our sample of 83 galaxies is divided into four mass quartiles with $7.3 \leqslant \log M / M_{\odot}<8.6,8.6 \leqslant \log M / M_{\odot}<9.0$, $9.0 \leqslant \log M / M_{\odot}<9.4,9.4 \leqslant \log M / M_{\odot}<10.2$. The stacked spectra are shown in Figure 2.

\subsection{Metallicity}

A few steps must be taken to infer metallicities from our stacked spectra. First, since [O III] and [O II] are widely separated in wavelength, a dust correction is required. We estimate extinction by measuring the Balmer decrement for each of the five galaxies in our $z<1.5$ subsample where both $\mathrm{H} \alpha$ and $\mathrm{H} \beta$ are detected (at $3 \sigma$ significance or greater). Consistent with our findings in Domínguez et al. (2013), we measure an average $\mathrm{H} \alpha / \mathrm{H} \beta=3.6$, with an rms of 1.1. We also stack all of the 28 galaxies with $1.3<z<1.5$; here we find $\mathrm{H} \alpha / \mathrm{H} \beta=2.48 \pm 0.33$, consistent with no dust. In the analysis that follows, we calculate line ratios assuming both no dust, and $\mathrm{H} \alpha / \mathrm{H} \beta=3.6 \pm 1.1$ (with a Calzetti et al. 2000 extinction curve). In the latter case, the scatter on the Balmer decrement is included in the metallicity errors.

Second, the flux measured from the $\mathrm{H} \beta$ emission line can be artificially reduced by stellar absorption, which is estimated to have an equivalent width (EW) of $3-4 \AA$ in similar grismselected galaxies (Domínguez et al. 2013). Since our stacked spectra only represent relative line fluxes, we estimate the $\mathrm{H} \beta$ EW from the mean [O III] EW of the individual stacked galaxies and $\left[\mathrm{O}{ }_{\mathrm{III}}\right] / \mathrm{H} \beta$ ratio for that stack. The values for the rest-frame $\mathrm{H} \beta$ EW range from 30 to $110 \AA$ (see Table 1 ). The relative $\mathrm{H} \beta$ fluxes are increased by $\left(\mathrm{EW}_{\text {rest }}(\mathrm{H} \beta)+3.5\right) / \mathrm{EW}_{\text {rest }}$ to account for this absorption.

Finally, $R_{23}$ is double-valued; a single measurement can correspond to either a high or low metallicity. Determining whether a galaxy lies on the high or low metallicity branch can be challenging (Kewley \& Ellison 2008; Henry et al. 2013).

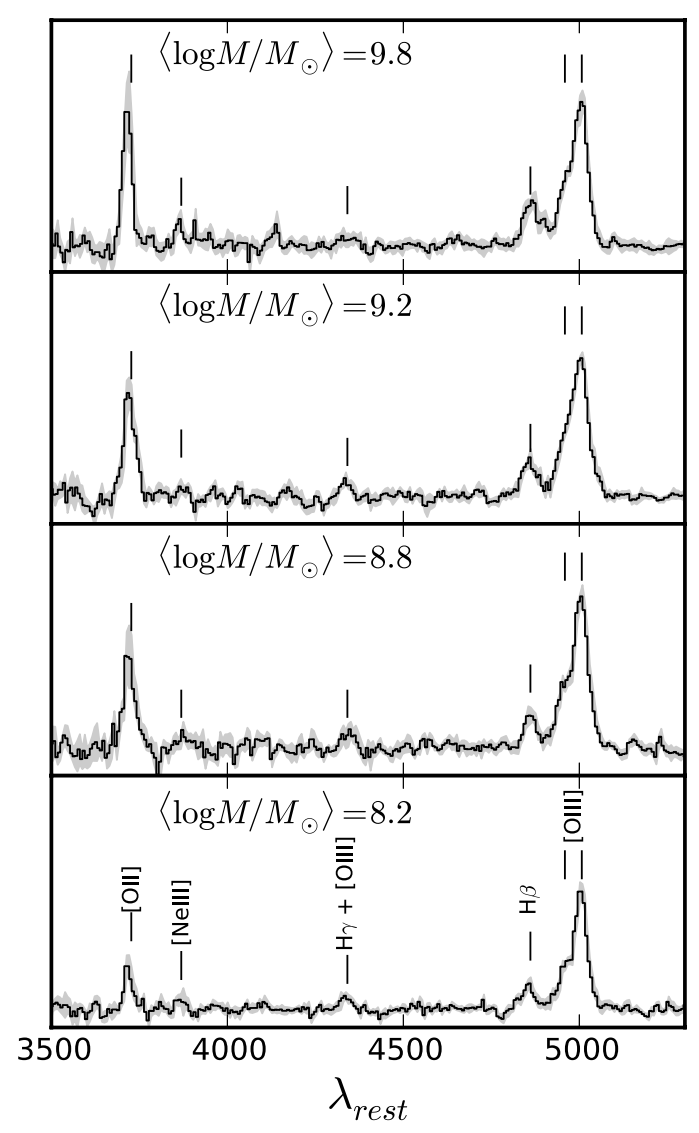

Figure 2. Stacked spectra are shown with shaded regions indicating the $\pm 1 \sigma$ uncertainty. Vertical lines mark the expected wavelengths of the line emission, including $\mathrm{H} \gamma+[\mathrm{O} \mathrm{III}] \lambda 4363$ and $[\mathrm{Ne}$ III $] \lambda 3869$. We do not interpret these weaker features because of their low $\mathrm{S} / \mathrm{N}$ and underlying stellar absorption.

Nevertheless, under the reasonable assumption that metallicity decreases toward lower masses, an average trend is expected: as mass decreases, $R_{23}$ should increase to $\log R_{23} \sim 1$, before turning over and decreasing. For the first time at high redshift, this trend is observed in Figure 3. We conclude that the $M=10^{8.8} M_{\odot}$ bin marks the "turn-around," with the two higher mass bins falling on the upper branch of $R_{23}$ and the lowest mass bin implying lower-branch metallicities. The middle panel of Figure 3 supports this conclusion; as in the local universe, the lowest metallicity galaxies have more highly ionized gas, as indicated by higher $\mathrm{O} 32(\log [\mathrm{O} \mathrm{III}] /[\mathrm{O} \mathrm{II}])$.

We have tested that the results from stacking are robust to scatter between the upper and lower-branch bins. Taking a simulated MZ relation with the same slope as we have measured, we adopt an intrinsic metallicity scatter of 0.1 dex (as at $z \sim 0.1$; Tremonti et al. 2004) and 0.2 dex of scatter in stellar mass due to our typical measurement error. We find that fewer than $10 \%$ of the galaxies in the bins adjacent to the turn-around should be attributed to the opposite $R_{23}$ branch, and less than $1 \%$ of galaxies in our high-mass bin will fall on the lower branch. This exercise shows that metallicities may be overestimated by $0.03-0.04$ dex in the $M=10^{9.2} M_{\odot}$ bin, and underestimated by the same amount in our lowest mass bin. We do not apply a correction for this effect, since its amplitude is smaller than our uncertainties.

Finally, as detailed in Kewley \& Ellison (2008), different metallicity calibrations of strong-line ratios yield systematically different abundances. In Table 1, metallicities are reported on 
Table 1

Measurements from Stacked Spectra

\begin{tabular}{|c|c|c|c|c|c|c|c|c|c|c|}
\hline $\begin{array}{l}\log M_{\star} / M_{\odot} \\
\text { (1) }\end{array}$ & $N$ & $\begin{array}{l}\langle z\rangle \\
(3)\end{array}$ & $\begin{array}{c}\mathrm{EW}(\mathrm{H} \beta) \\
(\AA) \\
(4)\end{array}$ & $\begin{array}{c}\log [\mathrm{O} I I I] / H \beta \\
\text { (5) }\end{array}$ & $\begin{array}{l}\mathrm{O} 32 \\
(6)\end{array}$ & $\begin{array}{c}\log R_{23} \\
\text { (7) }\end{array}$ & $\begin{array}{c}12+\log (\mathrm{O} / \mathrm{H}) \\
(\mathrm{KK} 04) \\
(8)\end{array}$ & $\begin{array}{c}12+\log (\mathrm{O} / \mathrm{H}) \\
\text { (Maiolino) } \\
(9)\end{array}$ & $\begin{array}{c}\mathrm{SFR} \\
\left(M_{\odot} \mathrm{yr}^{-1}\right) \\
(10)\end{array}$ & $\begin{array}{c}12+\log (\mathrm{O} / \mathrm{H}) \\
(\mathrm{FMR}) \\
(11)\end{array}$ \\
\hline \multicolumn{11}{|c|}{ Dust Corrected: $\mathrm{H} \alpha / \mathrm{H} \beta=3.6 \pm 1.1$} \\
\hline 8.2 & 21 & 1.82 & 110 & $0.77 \pm 0.09$ & $0.65 \pm 0.15$ & $0.85 \pm 0.09$ & $8.16_{-0.15}^{+0.16}$ & $7.59_{-0.16}^{+0.44}$ & 7 & $\cdots$ \\
\hline 8.8 & 22 & 1.73 & 47 & $0.81 \pm 0.08$ & $0.33 \pm 0.14$ & $0.98 \pm 0.08$ & $8.43_{-0.11}^{+0.16}$ & $8.03_{-0.33}^{+0.33}$ & 9 & 8.17 \\
\hline 9.2 & 21 & 1.77 & 36 & $0.66 \pm 0.06$ & $0.24 \pm 0.12$ & $0.85 \pm 0.07$ & $8.65_{-0.11}^{+0.14}$ & $8.47_{-0.26}^{+0.14}$ & 13 & 8.33 \\
\hline 9.8 & 19 & 1.74 & 29 & $0.53 \pm 0.07$ & $0.22 \pm 0.13$ & $0.74 \pm 0.08$ & $8.82_{-0.10}^{+0.08}$ & $8.68_{-0.12}^{+0.09}$ & 21 & 8.60 \\
\hline \multicolumn{11}{|c|}{ No Dust Correction } \\
\hline 8.2 & 21 & 1.82 & 110 & $0.76 \pm 0.09$ & $0.76 \pm 0.10$ & $0.84 \pm 0.09$ & $8.12_{-0.14}^{+0.15}$ & $7.57_{-0.15}^{+0.37}$ & 3 & $\cdots$ \\
\hline 8.8 & 22 & 1.73 & 47 & $0.82 \pm 0.08$ & $0.45 \pm 0.08$ & $0.96 \pm 0.08$ & $8.42_{-0.16}^{+0.30}$ & $8.03_{-0.38}^{+0.38}$ & 4 & 8.22 \\
\hline 9.2 & 21 & 1.77 & 36 & $0.67 \pm 0.06$ & $0.35 \pm 0.05$ & $0.83 \pm 0.06$ & $8.70_{-0.11}^{+0.09}$ & $8.53_{-0.16}^{+0.11}$ & 6 & 8.39 \\
\hline 9.8 & 19 & 1.74 & 29 & $0.54 \pm 0.07$ & $0.33 \pm 0.08$ & $0.71 \pm 0.07$ & $8.86_{-0.08}^{+0.06}$ & $8.72_{-0.09}^{+0.08}$ & 9 & 8.67 \\
\hline
\end{tabular}

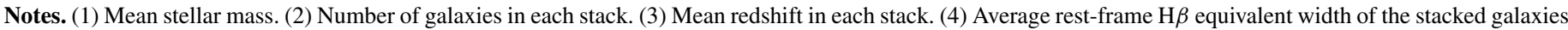

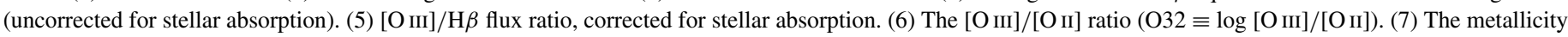

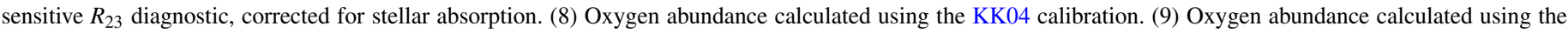

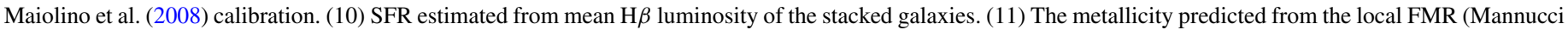
et al. 2011; given for the $M \gtrsim 10^{8.5} M_{\odot}$ mass bins where the FMR is defined.)

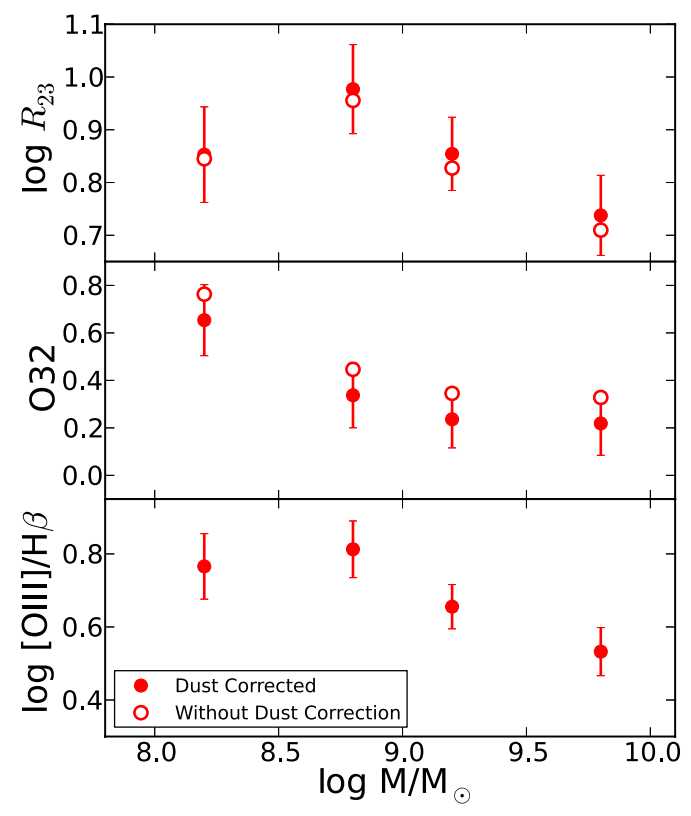

Figure 3. Emission line ratios measured from our stacked spectra (with and without dust correction) show that the turn-around metallicity is reached around $M \sim 10^{8.8} M_{\odot}$.

(A color version of this figure is available in the online journal.)

both the Kobulnicky \& Kewley (2004, KK04) and Maiolino et al. (2008) scale.

\section{STAR-FORMATION, SAMPLE BIAS, AND THE FUNDAMENTAL METALLICITY RELATION}

In the local universe, metallicity, stellar mass, and star formation rate (SFR) form a plane called the Fundamental Metallicity Relation (FMR; Mannucci et al. 2010), in the sense that galaxies with high specific SFRs have lower metallicities. As a result, it is important to consider selection effects when interpreting the $\mathrm{MZ}$ relation. For example, UV-continuum or emission-line selected samples may be biased toward higher SFRs and lower metallicities than mass-selected samples. Therefore, we use $\mathrm{H} \beta$ luminosity (measured analogously to the $\mathrm{H} \beta \mathrm{EW}$, using the mean $[\mathrm{O}$ III $]$ luminosity and the $[\mathrm{O} \mathrm{III}] / \mathrm{H} \beta$ ratio) to estimate the SFR for each stack. The $\mathrm{H} \beta$ luminosity is corrected for dust and stellar absorption, and multiplied by 2.86 to obtain the $\mathrm{H} \alpha$ luminosity. We then calculate the SFR from the relation in Kennicutt (1998), converted to a Chabrier (2003) IMF. ${ }^{13}$ In Table 1 we list the average SFR in each mass bin. Figure 4 shows that our dust-corrected SFRs lie very close to an extrapolation of the $z=1.76$ star-forming main sequence from Whitaker et al. (2012). This comparison shows that our grism-selected sample is not likely biased toward high specific SFRs.

Remarkably, Mannucci et al. (2010) report that the FMR does not evolve with redshift (at least out to $z \sim 3$ ). Further studies confirm this claim for smaller samples of low mass galaxies (Henry et al. 2013; Belli et al. 2013). Using our stack-averaged measurements, we can also test for evolution. In Table 1 we list the oxygen abundance, predicted from the FMR in Mannucci et al. (2011). This quantity shows excellent agreement with the metallicities calculated on the Maiolino et al. (2008) calibration (on which the FMR is defined). We conclude that our sample shows no evidence for evolution of the FMR.

\section{THE MASS-METALLICITY RELATION}

Figure 4 shows the MZ relation derived from our stacked spectra, for both the dust corrected and uncorrected measurements. It is clear that the dust correction represents only a small adjustment. Excluding our lowest mass bin, our (dust corrected) metallicities are $0.33,0.17$, and 0.10 dex lower than the Sloan Digital Sky Survey (SDSS) $z \sim 0.1$ measurement at $10^{8.8}, 10^{9.2}$, and $10^{9.8} M_{\odot}$ (Tremonti et al. 2004), suggesting more significant evolution in low mass galaxies. A similar trend has been observed in the intermediate-redshift MZ relation (also shown in

\footnotetext{
13 The SFR calibration in Kennicutt (1998) is defined for solar metallicity, and will therefore overestimate SFRs at low metallicity (by $25 \%$ and $37 \%$ for continuous star-forming, BC03 models with a Chabrier 2003 IMF at $Z=0.4 Z_{\odot}$ and $\left.Z=0.2 Z_{\odot}\right)$. However, in order to compare to previous measurements of the star-forming main sequence, we report SFRs for solar metallicity.
} 

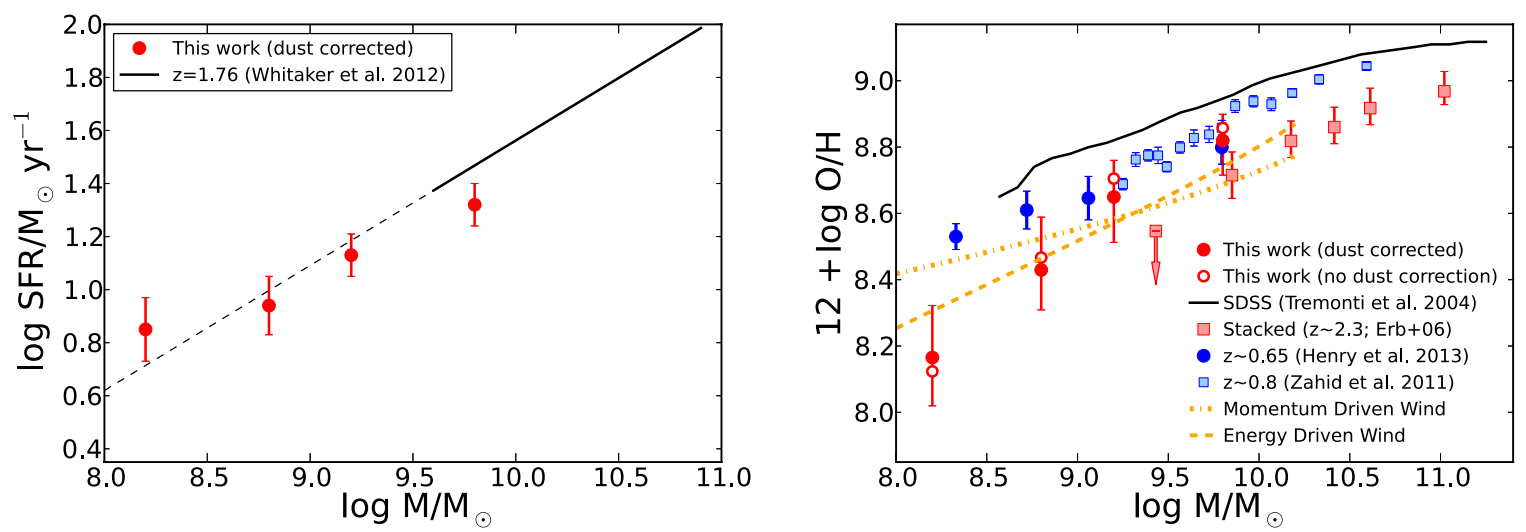

Figure 4. Left: the dust-corrected SFRs for our stacked spectra fall along an extrapolation of the $z=1.76$ star-forming main sequence, indicated by the dashed line. Error bars are derived from the error on the mean $\mathrm{H} \beta$ luminosity, and do not include the uncertainties associated with extinction correction. Right: the MZ relation at $z \sim 1.76$ declines steeply toward low stellar masses. All metallicities are given on the KK04 calibration (when necessary, converted using Kewley \& Ellison 2008). Intermediate redshift data from Zahid et al. (2011) and Henry et al. (2013) are binned averages. Orange curves (arbitrarily normalized) show how different prescriptions for galactic outflows change the slope of the MZ relation.

(A color version of this figure is available in the online journal.)

Figure 4; Zahid et al. 2011; Henry et al. 2013). In fact, in Henry et al. (2013) we interpreted this evolution as evidence for downsizing, where higher mass galaxies evolve onto the local MZ relation at earlier times. While the uncertainty on the evolution measured by the present data remains large, it is consistent with downsizing. Stacking larger samples of grism spectra can help to reduce the uncertainties, but, at the same time, robust measurements of the local MZ relation are needed for $M \lesssim 10^{8.5} M_{\odot}$. Comparison to local dwarf galaxies remains difficult, as most have metallicities derived directly from electron temperatures (which differ systematically from strong-line metallicities), or suffer from uncertainties regarding the $R_{23}$ branch (Berg et al. 2012; Ly et al. 2013).

We also compare our MZ relation to the measurement from $z \sim 2.3$ galaxies reported by Erb et al. (2006). These metallicities were measured from the $[\mathrm{N}$ II] $\lambda 6583 / \mathrm{H} \alpha$ ratio in stacked spectra (using the Pettini \& Pagel 2004 calibration), and are converted to KK04 metallicities using Kewley \& Ellison (2008). Figure 4 shows that our MZ relation agrees with the results from Erb et al. The somewhat higher metallicities in our data are consistent with the lower redshift $(z \sim 1.76)$ of our sample. The broad agreement between these measurements suggests that the systematic offsets between metallicity calibrations (which we removed) are not drastically different at low and high redshifts. Finally, while some low mass lensed galaxies have metallicity measurements at these redshifts (e.g., Wuyts et al. 2012), large scatter and many weak upper limits preclude meaningful comparisons.

Finally, we compare our data to theoretical models for the MZ relation. Following our implementation in Henry et al. (2013), we show two models calculated from the prescriptions in Davé et al. (2012). Figure 4 shows that a model regulated by momentum-driven winds predicts a shallower MZ slope than an energy-driven wind model. While we showed in Henry et al. (2013) that the intermediate redshift MZ relation is reproduced by momentum-driven winds, the higher redshift relation suggests evolution, as it more closely follows the energy-driven wind model. It is unlikely that a different choice of metallicity calibration would flatten our observed MZ relation to bring it closer in line with momentum-driven winds; among the widely used strong-line metallicity calibrations, only Pilyugin (2001) and Pilyugin \& Thuan (2005) yield MZ relations with significantly shallower slopes compared to a KK04 MZ relation. However, these shallow slopes may be inaccurate, owing to a saturation of the [O III] $\lambda 4363$ diagnostic at high metallicities (Kewley \& Ellison 2008). Nevertheless, the metallicity errors remain large, so while our data favor energy-driven winds, we cannot rule out the shallower slope associated with momentum-driven winds.

We have presented the first measurement of the MZ relation from a large sample of low mass, high redshift galaxies. Although emission-line selected, these galaxies are not extreme in their SFRs or metallicities. Rather, our results show excellent agreement with extrapolations of the star-forming main sequence and $\mathrm{MZ}$ relation at similar redshifts. We conclude that the low-mass, high-redshift MZ relation suggests a downsizing evolution and a preference for feedback from energy-driven winds.

We acknowledge Dawn Erb, Kate Whitaker and Danielle Berg for helpful discussions. This research was supported by an appointment to the NASA Postdoctoral Program at the Goddard Space Flight Center, administered by Oak Ridge Associated Universities through a contract with NASA. A.H. also acknowledges support from HST GO 11696, 12284, and 12568.

\section{REFERENCES}

Atek, H., Malkan, M., McCarthy, P., et al. 2010, ApJ, 723, 104 Atek, H., Siana, B., Scarlata, C., et al. 2011, ApJ, 743, 121 Bedregal, A., Scarlata, C., Henry, A., et al. 2013, arXiv:1309.3567 Belli, S., Jones, T., Ellis, R. S., \& Richard, J. 2013, ApJ, 722, 141 Berg, D. A., Skillman, E. D., Marble, A. R., et al. 2012, ApJ, 754, 98 Bertin, E., \& Arnouts, S. 1996, A\&A, 117, 393 Brammer, G. B., Sánchez-Janssen, R., Labbé, I., et al. 2012, ApJL, 758, L17 Brooks, A., Governato, F., Booth, C. M., et al. 2007, ApJL, 655, 17L Bruzual, G., \& Charlot, S. 2003, MNRAS, 344, 1000

Calzetti, D., Armus, L., Bohlin, R. C., et al. 2000, ApJ, 533, 682

Casertano, S., de Mello, D., Dickinson, M., et al. 2000, AJ, 120, 2747 Chabrier, G. 2003, PASP, 115, 763

Colbert, J. W., Teplitz, H., Atek, H., et al. 2013, arXiv:1305.1399 Dalcanton, J. J. 2007, ApJ, 658, 941

Davé, R., Finlator, K., \& Oppenheimer, B. D. 2012, MNRAS, 421, 98 Domínguez, A., Siana, B., Henry, A., et al. 2013, ApJ, 763, 145 Erb, D. K. 2008, ApJ, 674, 151

Erb, D. K., Shapley, A. E., Pettini, M., et al. 2006, ApJ, 644, 813 Finlator, K., \& Davé, R. 2008, MNRAS, 385, 2181

Hainline, K., Shapley, A. E., Kornei, K. A., et al. 2009, ApJ, 701, 52 Hayashi, M., Motohara, K., Shimasaku, K., et al. 2009, ApJ, 691, 140 
Henry, A., Martin, C. L., Finlator, K., \& Dressler, A. 2013, ApJ, 769, 148 Illingworth, G. D., Magee, D., Oesch, P. A., et al. 2013, arXiv:1305.1931

Juneau, S., Dickinson, M., Alexander, D. M., \& Salim, S. 2011, ApJ, 736, 104

Kennicutt, R. C. 1998, ARA\&A, 36, 189

Kewley, L. J., \& Ellison, S. L. 2008, ApJ, 681, 1183

Kobulnicky, H. A., \& Kewley, L. J. 2004, ApJ, 617, 240 [KK04]

Kriek, M., van Dokkum, P. G., Labbé, I., et al. 2009, ApJ, 700, 221

Liu, X., Shapley, A. E., Coil, A. L., Brinchmann, J., \& Ma, C.-P. 2008, ApJ, 678,758

Ly, C., Malkan, M. A., Nagao, T., et al. 2013, arXiv:1307.7712

MacKenty, J. W., Kimble, R. A., O’Connell, R. W., \& Townsend, J. A. 2010, Proc. SPIE, 7731, $77310 Z$

Maiolino, R., Nagao, T., Grazian, A., et al. 2008, A\&A, 488, 463

Mannucci, F., Cresci, G., Maiolino, R., Marconi, A., \& Generucci, A. 2010, MNRAS, 408, 2115

Mannucci, F., Salvaterra, R., \& Campisi, M. A. 2011, MNRAS, 414, 1263

Pagel, B. E. J., Edmunds, M. G., Blackwell, D. E., Chun, M. S., \& Smith, G. 1979, MNRAS, 189, 95
Peeples, M. S., \& Shankar, F. 2011, MNRAS, 417, 2962

Pettini, M., \& Pagel, B. E. J. 2004, MNRAS, 384, 59

Pilyugin, L. S. 2001, A\&A, 374, 412

Pilyugin, L. S., \& Thuan, T. X. 2005, ApJ, 631, 231

Shapley, A. E., Coil, A. L., Ma, C.-P., \& Bundy, K. 2005, ApJ, 635, 1006

Simcoe, R. A., Metzger, M. R., Small, T. A., \& Araya, G. 2000, BAAS 32,758

Teplitz, H. I., McLean, I. S., Becklin, E. E., et al. 2000, ApJ, 533, 65

Tremonti, C. A., Heckman, T. M., Kauffmann, G., et al. 2004, ApJ, 613,898

Trump, J. R., Konidaris, N. P., Barro, G., et al. 2013, ApJL, 763, L6

Whitaker, K. E., van Dokkum, P. G., Brammer, G., \& Franx, M. 2012, ApJL, 754, 29L

Wright, S. A., Larkin, J. E., \& Law, D. R. 2009, ApJ, 699, 421

Wuyts, E., Rigby, J. R., Sharon, K., \& Gladders, M. 2012, ApJ, 755, 73

Xue, Y. Q., Luo, B., Brandt, W. N., et al. 2011, ApJS, 195, 10

Yuan, T.-T., Kewley, L. J., \& Richard, J. 2013, ApJ, 763, 9

Zahid, H. J., Kewley, L. J., \& Bresolin, F. 2011, ApJ, 730, 137 